\title{
FIRST COUNTABLE HYPERSPACES
}

\author{
R. E. SMITHSON
}

\begin{abstract}
An example is given which shows that the space of compact subsets $\mathscr{K}(X)$ of a first countable space $X$ need not be first countable in the finite topology. Further, it is shown that if $\nVdash(X)$ is first countable then each compact subset of $X$ is separable. Finally a characterization of $\mathcal{K}(X)$ first countable in terms of a weak second countability condition is derived.
\end{abstract}

1. Introduction. Let $X$ be a topological space and let $\mathscr{K}(X)$ be the set of nonempty compact subsets of $X$. If $U_{1}, \ldots, U_{n}$ are open subsets of $X$, then a base for a topology on $\mathcal{K}(X)$ is generated by sets of the form $\left\langle U_{1}, \ldots, U_{n}\right\rangle$ $=\left\{K \in \mathcal{K}(X): K \subset \cup_{i=1}^{n} U_{n}, K \cap U_{i} \neq \varnothing, i=1, \ldots, n\right\}$. This topology is called the finite topology and was studied extensively by Michael [1]. (In [1] the topology was studied for the set of nonempty closed subsets and the nonempty subsets of $X$ as well as $\mathscr{K}(X)$.) In [1] Michael asserted that a space $X$ is first countable if and only if $\mathcal{K}(X)$ is first countable (Proposition 4.5, Part 3). It is easy to see that if $\mathcal{K}(X)$ is first countable,then so is $X$. However, the purpose of this note is to show that $X$ first countable does not imply that $\mathcal{K}(X)$ is first countable. In $\$ 2$ we give an example of a first countable, compact, Hausdorff space $X$ such that $\mathscr{K}(X)$ is not first countable. Next we prove a theorem which gives a necessary condition for $\mathscr{K}(X)$ to be first countable. Then we derive a characterization of spaces in which $\mathscr{K}(X)$ is first countable based on the basic open sets defined above for the finite topology and on a countability condition on $X$. Finally we introduce another countability condition and ask some related questions.

2. First countable hyperspaces. Before presenting the example we need the following lemma.

Lemma 1. Let $\mathcal{Q}=\left\langle U_{1}, \ldots, U_{n}\right\rangle$ and $\mathfrak{V}=\left\langle V_{1}, \ldots, V_{k}\right\rangle$ be two basic open sets in the finite topology on $\mathcal{K}(X)$. Then $\mathcal{V} \subset$ Q 1 if and only if for each $U_{i}$ there is a $V_{j}$ such that $V_{j} \subset U_{i}$ and $\cup_{j=1}^{k} V_{j} \subset \cup_{i=1}^{n} U_{i}$.

Proof. First suppose $\mathscr{V} \subset \mathcal{Q}$. Then $\cup_{j=1}^{k} V_{j} \subset \cup_{i=1}^{n} U_{i}$. Otherwise let $x_{j} \in V_{j}$ for $i=1, \ldots, k$ and $x_{k+1}$ be a point of $\cup_{j=1}^{k} V_{j}$ which is not in $\cup_{i=1}^{n} U_{i}$. Then $\left\{x_{j}: j=1, \ldots, k+1\right\} \in \mathscr{V} \backslash Q_{1}$. Next suppose that there is a $U_{i}$ such that $V_{j} \backslash U_{i} \neq \varnothing$ all $j=1, \ldots, k$ and let $x_{j} \in V_{j} \backslash U_{i}$. Then $\left\{x_{j}\right.$ : $j=1, \ldots, k\} \in \mathcal{V} \backslash \mathcal{Q}$. Hence, for each $U_{i}$, there is a $V_{j}$ such that $V_{j} \subset U_{i}$.

Received by the editors April 28, 1975 and, in revised form, June 25, 1975.

AMS (MOS) subject classifications (1970). Primary 54B20.

Key words and phrases. First countable, separable, hyperspaces, the finite topology, second countability. 
Now to prove the converse let $K \in \mathcal{K}(X)$ and $K \in \mathcal{V}$. Then $K \subset$ $\cup_{j=1}^{k} V_{j}$ and thus $K \subset \cup_{i=1}^{n} U_{i}$. Further, let $V_{j}$ be such that $V_{j} \subset U_{i}$. Then, since $K \cap V_{j} \neq \varnothing, K \cap U_{i} \neq \varnothing$. Hence, $K \cap U_{i} \neq \varnothing$ for all $i$ and $K \in \mathcal{Q}$. Then $\mathfrak{V} \subset$ Q .

The space used in the following example is Example 97 in [2].

Example. Let $X$ consist of two concentric circles $C_{1}, C_{2}$ in the plane. Assume that $C_{2}$ is the outer circle. A subbase for the topology on $X$ is constructed as follows. First let each singleton in $C_{2}$ be open. Then take an open interval of $C_{1}$ together with all of its radial projection on $C_{2}$ except the midpoint of the projection.

With this topology $X$ is first countable, Hausdorff and compact. Furthermore, if $K$ is a closed (proper) subinterval of $C_{1}$ together with its radial projection, then $K$ is compact. Let $\left(k_{1}, k_{2}\right),\left(k_{3}, k_{4}\right), \ldots,\left(k_{n-1}, k_{n}\right)$ be a finite set of intervals of $C_{1}$ which cover $K \cap C_{1}$. Let $c_{1}, \ldots, c_{n}$ be the midpoints of the projections of the intervals $\left(k_{i-1}, k_{i}\right)$. Finally let $a, b$ be the endpoints of $K \cap C_{2}$ and $U_{1}, \ldots, U_{n}$ be the open sets generated by the intervals $\left(k_{i-1}, k_{i}\right)$. Then Ql $=\left\langle U_{1}, \ldots, U_{n},\left\{c_{1}\right\}, \ldots,\left\{c_{n}\right\},\{a\},\{b\}\right\rangle$ is an open set containing $K$ when $c_{i} \in K$. Next if $\widetilde{\mathcal{C}} \subset \mathcal{Q}$, and if $\mathfrak{\mathcal { V }}=\left\langle V_{1}, \ldots, V_{t}\right\rangle$, then for each singleton $\left\{c_{i}\right\}$ there must be a $V_{j}$ such that $\left\{c_{i}\right\}=V_{j}$. Since $K \cap C_{2}$ is uncountable, there are an uncountable number of ways of constructing the set $\mathcal{Q}$ with different singleton sets. Thus there cannot be a countable basis for the neighborhoods of $K$ in $\mathscr{K}(X)$.

Next we give the theorem referred to earlier.

THEOREM 2. Let $X$ be such that each compact subset is regular. If ih $(X)$ is first countable, then each compact subspace of $X$ is separable (in the relative topology).

Proof. Suppose that $\mathscr{K}(X)$ is first countable and let $K \subset X$ be compact. Let $\mathscr{G}(K)$ be a countable base for the neighborhoods of $K$ in $\mathscr{K}(X)$. We may assume a member $B_{k}$ of $\mathscr{B}(K)$ is of the form $\left\langle U_{1}^{k}, \ldots, U_{n_{k}}^{k}\right\rangle$ where $U_{i}^{k}$ is open in $X$. Assume $k$ is fixed and pick a point $a_{k, i} \in U_{i}^{k^{k}} \cap K$ for each $i=1, \ldots, n_{k}$, and set $A_{k}=\left\{a_{k, i}: i=1, \ldots, n_{k}\right\}$. Now set $A=\cup_{k=1}^{\infty} A_{k}$. We claim that $A$ is dense in $K$. For let $x \in K$ and let $U$ be a $K$-open set containing $x$. Let $V$ be a $K$-open set such that $x \in V \subset V^{*} \subset U$ where $V^{*}$ denotes the closure of $V$ (in $K$ ). Then there exist open sets (in $X$ ) $W_{1}$ and $W_{2}$ such that $U=K \cap W_{1}$ and $K \backslash V^{*}=K \cap W_{2}$. Since $K \in\left\langle W_{1}, W_{2}\right\rangle$ there is a member $B_{k}$ of $\mathscr{B}(K)$ such that $K \in B_{k} \subset\left\langle W_{1}, W_{2}\right\rangle$ and hence, by Lemma 1 a set $U_{i}^{k} \subset W_{1}$. Thus $a_{k, i} \in U$. Hence, $A$ is dense in $K$ and therefore each compact subset of $X$ is separable.

REMARK. Theorem 2 could have been used to show that $K(X)$ need not be first countable whenever $X$ is. In fact Theorem 2 could have been applied in the above example.

Now by means of the following definitions, we give a characterization for $\mathcal{K}(X)$ to be first countable. In this definition a proper cover of a set $A$ is a cover $\mathcal{Q}$ such that $U \in \mathcal{Q}$ implies that $U \cap A \neq \varnothing$.

Definition. A topological space $X$ is called compactly second countable if and only if for each compact set $K \subset X$ there is a countable collection of open sets $\Re$ so that whenever $\left\{U_{1}, \ldots, U_{n}\right\}$ is a proper cover of $K$ there is a 
proper cover $\left\{V_{1}, \ldots, V_{m}\right\} \subset \mathscr{B}$ of $K$ such that $\cup_{j=1}^{m} V_{j} \subset \cup_{i=1}^{n} U_{i}$ and for each $U_{i}$, there is a $V_{j}$ with $V_{j} \subset U_{i}$.

THEOREM 3. A space $X$ is compactly second countable if and only if $\operatorname{th}(X)$ is first countable.

Proof. Let $X$ be compactly second countable and let $K \in \mathcal{K}(X)$ with 9 the given countable collection of open sets. Let $\mathscr{T}(K)$ be the collection of open subsets of $\mathcal{K}(X)$ which are constructed from the finite proper covers of $K$ contained in $\mathscr{B}$. Then $\mathscr{B}(K)$ is countable. To see that it is a base for the neighborhoods of $K$ let $K \in\left\langle U_{1}, \ldots, U_{n}\right\rangle$. Then $\left\{U_{1}, \ldots, U_{n}\right\}$ is a proper cover of $K$. The definition of compactly second countable and Lemma 1 imply that there is a proper cover $\left\{V_{1}, \ldots, V_{m}\right\}$ of $K$ in 9 such that $\left\langle V_{1}, \ldots, V_{m}\right\rangle \subset\left\langle U_{1}, \ldots, U_{n}\right\rangle$. Thus $\mathscr{K}(X)$ is first countable.

Next let $\mathscr{K}(X)$ be first countable. Then for $K \in \mathscr{K}(X)$ there is a countable base $\mathscr{B}(K)$ for the neighborhoods of $K$. We may assume that each member $B_{k}$ of $\mathscr{B}(K)$ has the form $B_{k}=\left\langle U_{1}^{k}, \ldots, U_{n_{k}}^{k}\right\rangle$. Now let $\mathscr{B}=\left\{U_{i}^{k}\right.$ : $\left.k \geqslant 1,1 \leqslant i \leqslant n_{k}\right\}$. If $\left\{U_{1}, \ldots, U_{n}\right\}$ is a proper open cover of $K$, then $\left\langle U_{1}, \ldots, U_{n}\right\rangle$ is an open set containing $K$. Thus there is a $B_{k} \in \mathscr{B}(K)$ such that $B_{k} \subset\left\langle U_{1}, \ldots, U_{n}\right\rangle$ and $\left\{U_{1}^{k}, \ldots, U_{n_{k}}^{k}\right\}$ is the desired proper cover in 93 . Hence, $X$ is compactly second countable.

By combining Theorems 2 and 3 we obtain

COROllary 4. If $X$ is a compactly second countable, regular space, then each compact subset of $X$ is separable.

REMARK. If $X$ is compactly second countable, then each compact set is in a sense weakly second countable in the relative topology. But it does not appear to imply that a compact subspace is second countable. Moreover, the assumption that each compact subspace is second countable (in the relative topology) does not imply that $X$ is compactly second countable. For there is a subspace of the Tychonoff plank in which each compact set is metrizable but the space is not first countable. Thus we ask the following question.

Question 1. Under what conditions is a space whose compact sets are second countable compactly second countable?

Next we introduce another similar definition.

Definition. A space is called compactly first countable if and only if each compact subset has a countable base for its neighborhoods.

REMARK. It follows from the definitions that a compactly second countable space is compactly first countable. Hence, if $\mathscr{K}(X)$ is first countable, then $X$ is compactly first countable. Moreover, it is also straightforward to show

Proposition 5. If $X$ is metrizable, then $X$ is compactly first countable.

It is not known whether or not Proposition 5 can be extended to general first countable spaces. Thus we end with some questions.

Question 2. The example given above is a first countable space which is not compactly first countable. Thus what additional conditions are needed in order that a first countable space be compactly first countable?

Question 3. Under what conditions is $\mathscr{h}(X)$ first countable for a compactly first countable space? 


\section{REFERENCES}

1. E. Michael, Topologies on spaces of subsets, Trans. Amer. Math. Soc. 71 (1951), 152-182. MR 13, 54.

2. L. A. Steen and J. A. Seebach, Jr., Counterexamples in topology, Holt, Rinehart and Winston, New York, 1970. MR 42 \#1040.

Department of Mathematics, University of Wyoming, Laramie, Wyoming 82071 